\title{
INCLUSION OF WARM PRE-STRESS IN FRENCH RPV STRUCTURAL INTEGRITY ASSESSMENT
}

\author{
Dominique Moinereau ${ }^{1}$, Patrick Le Delliou ${ }^{1}$, Malik Ait-Bachir ${ }^{2}$, Stéphane Chapuliot $^{3}$, \\ Stéphane Marie ${ }^{3}$, Clémentine Jacquemoud ${ }^{4}$ and Benoit Tanguy ${ }^{4}$
}

${ }^{1}$ EDF Lab Les Renardières, Moret-sur-Loing, France

${ }^{2}$ EDF SEPTEN, Lyon, France

${ }^{3}$ AREVA NP, Paris La Défense, France

${ }^{4}$ CEA Saclay, Gif sur Yvette, France

\begin{abstract}
Evaluation of the fracture resistance of nuclear reactor pressure vessel (RPV) regarding the risk of brittle fracture is a key point in the structural integrity assessment of the component. Such approach is codified in French RSE-M code, based on a conservative methodology. With respect to long term operation, an improvement of the present methodology is in progress to reduce this conservatism, with possible inclusion of the warm pre-stress (WPS) concept in the assessment. After a short description of the WPS concept, the process engaged in France to allow inclusion of WPS in the integrity assessment is presented.

Experimental and numerical studies have been conducted in France (also including international collaborations and projects) to demonstrate and validate the beneficial effect of WPS on the brittle fracture resistance of RPV steels. A large panel of experimental results and data is now available obtained on small, medium and large scale specimens on representative RPV steels (including highly irradiated RPV materials). These data have been included in a specific WPS experimental database.

A new criterion - ACE criterion - has been proposed for an easy simplified evaluation of warm pre-stress effect on the brittle fracture resistance of RPV steels. Accuracy and conservatism of the criterion is verified by comparison to experimental results and numerical analyses.

Implementation of the WPS effect in the French RSE-M code (for in service assessment) is now available. The present paper summarizes all these steps leading to codification of WPS in RSE-M code.
\end{abstract}

\section{INTRODUCTION}

The justification of in-service integrity of French RPV is periodically revised and transmitted to French Safety Authority. In link with NPP life extension, an extensive work is conducted in France to gather necessary elements for constituting a RPV irradiated area (shell rings) assessment document beyond 40 years, referring a 60 years life justification. In the field of structural integrity assessment, engaged actions must enable to propose new validated methodologies with the aim to improve existing margins regarding RPV integrity justification. From a mechanical point of view, these methodologies mainly concern several aspects, such crack initiation and crack arrest in the brittle or brittle - ductile transition fracture domain, and size effect on fracture toughness properties.

The warm pre-stress (WPS) is one of the retained possibilities to improve RPV integrity assessment regarding the evaluation of risk of brittle fracture initiation. An extensive work has been conducted on this topic (WPS) by French organizations EDF, CEA and AREVA, including experimental, analytical and numerical aspects. The objective of such work program was to give sufficient elements to demonstrate, understand, model and validate the effect of WPS. Main actions are now achieved and clearly confirm the beneficial effect of WPS on the brittle fracture resistance of RPV steels: 
- an extensive experimental validation of WPS on current RPV steels, covering a wide range of experimental conditions (at small, medium or large scales): un-irradiated and irradiated materials, specimens and structures, uniaxial and biaxial loading, loading paths ...

- the constitution of one specific WPS database, gathering all available data and results

- a validation of analytical and numerical models devoted to the evaluation of fracture resistance

Based on this work, an analytical criterion - ACE criterion - is proposed for an easy analytical evaluation of fracture toughness $\mathrm{K}_{\text {frac }}$ induced by WPS type loading. Implementation of the WPS effect in the French RSE-M code (for in service assessment) is now completed.

This paper summarizes the process and the main steps leading to codification of WPS and effective use in the integrity assessment.

\section{THE WARM PRE-STRESS IN RPV ASSESSMENT}

Material fracture toughness $\mathrm{K}_{\mathrm{Ic}}$ (or $\mathrm{K}_{\mathrm{Ic}}$ ) is usually determined through monotonic loading in isothermal conditions (constant temperature) on standard compact tension (CT) specimens. Thus, based on high constraint geometry like CT specimens, conservative values of fracture toughness are obtained to be used in a classical RPV integrity assessment. However, for most cases, typical loading in a RPV assessment is more complex (e.g. pressurized thermal shock transient PTS), involving non monotonic loading on a large range of temperature.

In most of the Regulatory codes (including RSE-M [1]), the safety margins regarding brittle fracture of RPV are evaluated by the comparison between the material properties $\left(\mathrm{K}_{\mathrm{Ic}}\right.$ fracture toughness generally measured on CT specimens, blue curve on Figure 1) and the 'mechanical damage' of the component induced by the loading $\left(\mathrm{K}_{\mathrm{cp}}\right.$ or $\mathrm{K}_{\mathrm{J}}$ stress intensity factor, pink curve on Figure 1) (Figure 1).

The warm pre-stress (WPS) in RPV integrity assessment is well known to modify and improve the brittle fracture resistance of ferritic steels. A cooling PTS type transient generally involves several steps, as depicted on Figures 1 and 2:

- an initial preloading at 'high' temperature $\mathrm{T}_{1}$ with an increasing of stress intensity factor $\mathrm{K}$

- a decreasing $\mathrm{K}$ in a second step with decreasing temperature, between temperatures $\mathrm{T}_{1}$ and $\mathrm{T}_{2}$

- a possible final reloading at the lower temperature $\mathrm{T}_{2}$

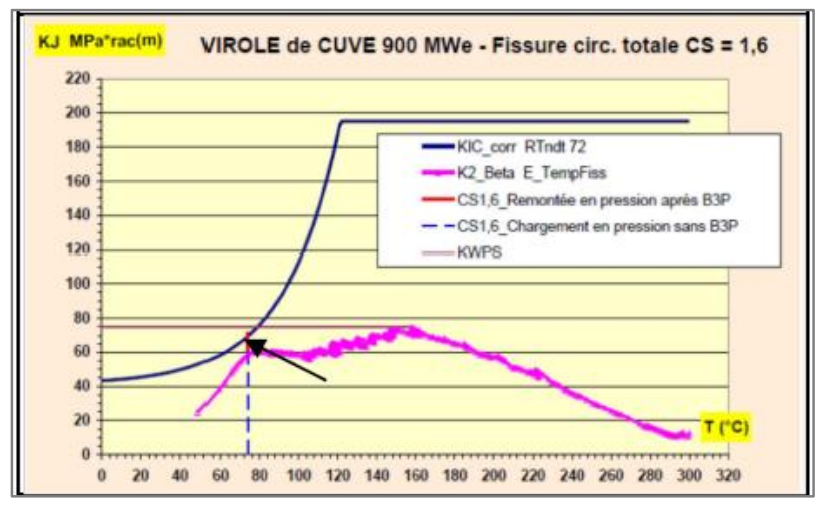

Figure 1. Evaluation of margins during a PTS transient regarding brittle fracture for a typical RPV PTS transient (comparison between $\mathrm{K}_{\mathrm{Ic}}$ and $\mathrm{K}_{\mathrm{J}}$ )

The consequences of the WPS effect, due to the load history, can be shortly summarized as follows:

- brittle fracture initiation is excluded - when cooling - during the unloading phase of $\mathrm{K}$ (also if the loading $K$ remains constant), even if the loading path $K_{J}-T$ intersects the $K_{I c}$ material fracture toughness lower bound curve (the 'conservative principle') 
- in case of an additional reloading at lower temperature $\left(\mathrm{T}_{2}\right)$, the brittle failure initiation would be generally obtained for a higher toughness, compared to the original material fracture toughness obtained on a 'virgin' material in isothermal conditions.
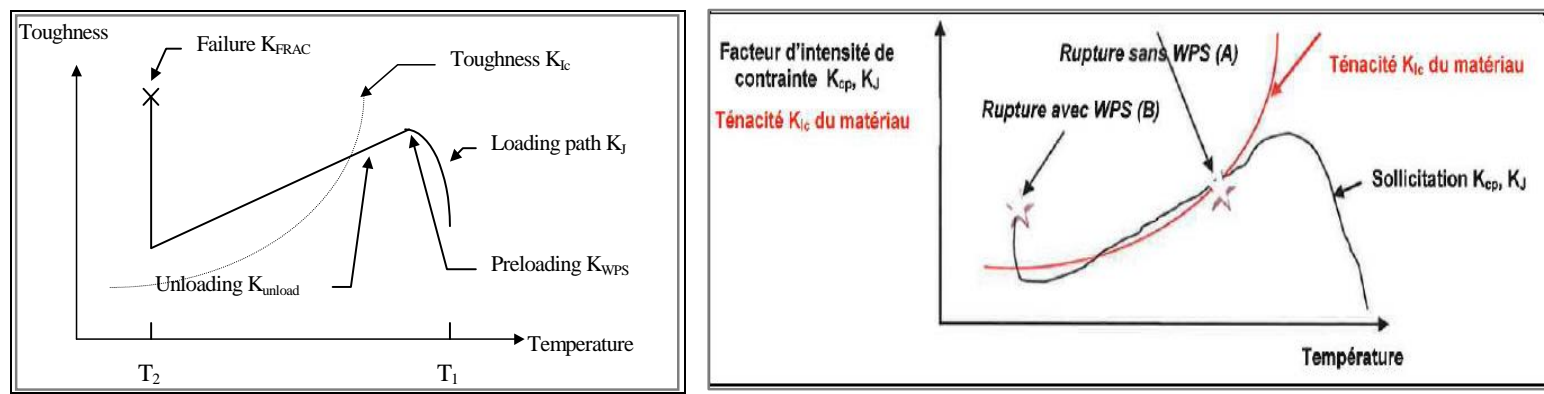

Figure 2. Warm pre-stress principle (comparison between fracture toughness $\mathrm{K}_{\mathrm{Ic}}$ and loading $\mathrm{K}_{\mathrm{cp}}$ or $\mathrm{K}_{\mathrm{J}}$ )

This effect of WPS is attributed to several factors, such as crack tip blunting, and for some WPS paths a compressive residual stress zone ahead of the crack tip ... An illustration of some typical WPS loading paths is shown on Figure 3.
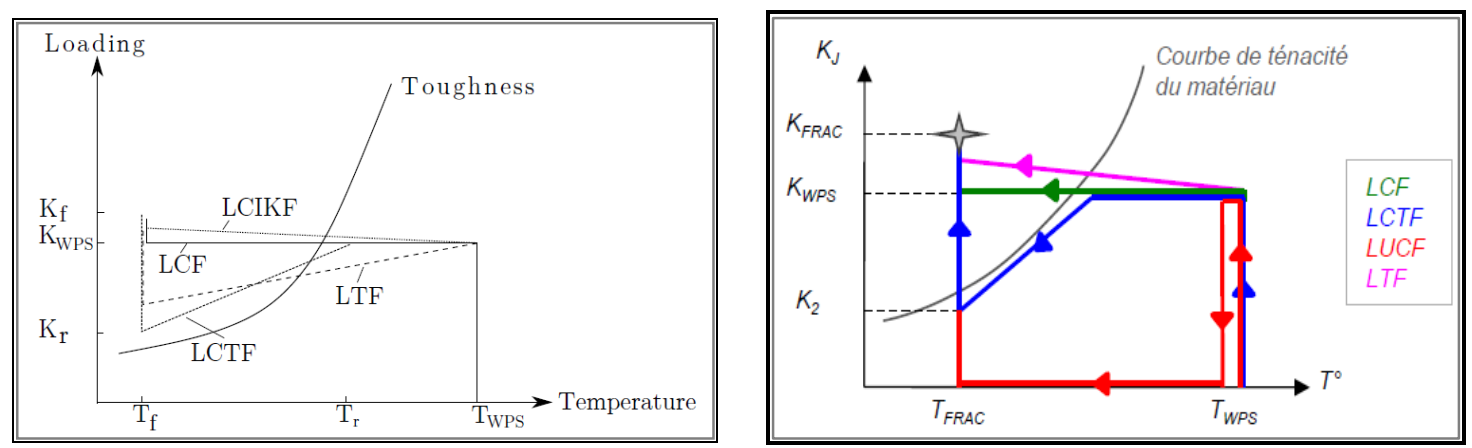

Figure 3. Illustration of typical WPS loading paths

This effect, well known and widely described in literature, is included in some Codes (e.g. ASME Section XI, KTA). It is not the case in French RSE-M code [1]: the assessment of a RPV subjected to PTS transient doesn't take into account the potential beneficial effect of the load history ('warm pre-stress WPS') on the vessel resistance regarding to the risk of brittle failure. In order to justify the introduction of this concept in French RPV assessment and Regulatory Code RSE-M, significant R\&D actions have been conducted by EDF, CEA and AREVA, - including also international collaborations with international partners - to demonstrate, model and validate the beneficial WPS effect on materials fully representative of RPV steels.

\section{PROCESS FOR VALIDATION OD WARM PRE-STRESS AND IMPLEMENTATION IN CODIFICATION}

In order to validate and codify the warm pre-stress effect in French RSE-M code, the present methodology has been used including the following aspects:

- an extensive experimental validation of WPS on representative RPV steels covering a wide range of experimental conditions (un-irradiated and irradiated steels, aged steels, various specimens and structures, loading path, complex loading ...)

- the development of a specific WPS database to gather all experimental data (experimental conditions and fracture results) and allow evaluation of WPS analytical and numerical models

- a proposal of an analytical engineering model for an easy evaluation of materials fracture toughness induced by WPS 
- the development of a more refined model based on local approach of fracture and requiring finite element computations ('modified' Beremin model) for a better understanding of fracture process during experiments and validation of analytical results

- a proposal of implementation of WPS in French RSE-M code

Each aspect of this methodology is summarized in the following parts of the document.

\section{EXPERIMENTAL VALIDATION OF WARM PRE-STRESS ON RPV STEELS}

Extensive R\&D programs have been conducted by French organizations to experimentally demonstrate the validity of warm pre-stress on the brittle fracture resistance of ferritic RPV steels (including international collaborations). A significant volume of experimental data is now available covering a wide range of experimental conditions:

- typical RPV steels: 16MND5 ( A508 Cl3), 18MND5 ( A533B)

- thermal aged steels for simulation of irradiation embrittlement (17MoV8.4 mod.)

- un-irradiated and irradiated RPV steels (16MND5), including highly irradiated materials (3.5 $10^{19}$ $\mathrm{n} / \mathrm{cm}^{2}<$ fluence $\Phi<\sim 1.410^{20} \mathrm{n} / \mathrm{cm}^{2}$ ), coming both from EDF Irradiation Surveillance Program (PSI) and Materials Testing Reactors (MTR)

- uniaxial or biaxial loading with mechanical or thermal loading

- fracture mechanics specimens (CT, SENB, CTpor ...)

- medium or large scale specimens (cylinder, PTS-D disks, cruciform specimens, bend bars ..)

- $\quad$ WPS type loading path (Figure 3) : LCF, LUCF, LTF, LCTF, LCIKF ...

$\circ$ LCF : Load Cooling Fracture

- LUCF : Load Unload Cooling Fracture

- LTF : Load Transient Fracture

- LCTF : Load Cooling Transient Fracture

○ LCIKF : Load Cooling Increasing K Fracture

- $\quad$ WPS experimental loading conditions : $\mathrm{K}_{\mathrm{WPS}}, \mathrm{T}_{\mathrm{WPS}}, \mathrm{K}_{\text {unload }}\left(\right.$ or $\left.\mathrm{K}_{2}\right), \mathrm{T}_{\mathrm{Frac}}, \mathrm{K}_{\mathrm{FRAC}} \ldots$

Numerous reports and publications are available, given all corresponding details and results (e.g. [2] to [10]). Based on these experiments, the beneficial effect of WPS regarding the brittle fracture resistance of RPV steels is fully confirmed, whatever the experimental conditions (e.g. Figures 4 to 9):

- the 'conservative principle' is again always verified, no fracture occurs during the cooling phase even if $\mathrm{K}_{\mathrm{J}}$ curve crosses the $\mathrm{K}_{\mathrm{Ic}}$ fracture toughness curve, with constant (e.g. LCF) or decreasing $\mathrm{K}_{\mathrm{J}}(\mathrm{e}$.g. LTF, LCTF)

- an increase of the brittle fracture resistance of the material ( $\left.\mathrm{K}_{\mathrm{FRAC}}\right)$ is generally observed in case of final reloading at low temperature, compared to the initial isothermal fracture toughness

This beneficial effect of WPS on the fracture resistance of the material can be shortly illustrated by some few significant results on the following figures.

WPS large scale experiment: MPA SMILE cylinder under PTS transient (17MoV8.4 mod.) [2]

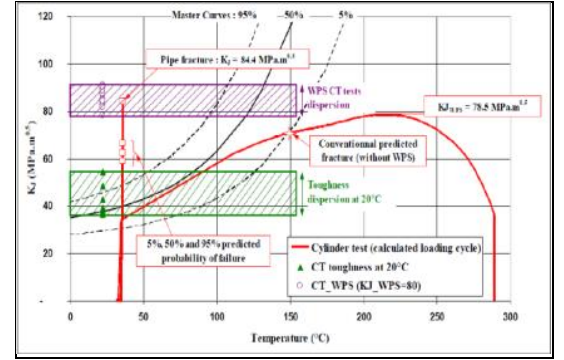

Figure 4. WPS large scale experiment on MPA SMILE cylinder (PTS transient) 


\section{Influence of a biaxial loading on WPS: NESC VII CEA cruciform specimens (18MND5) [3][5][6]}
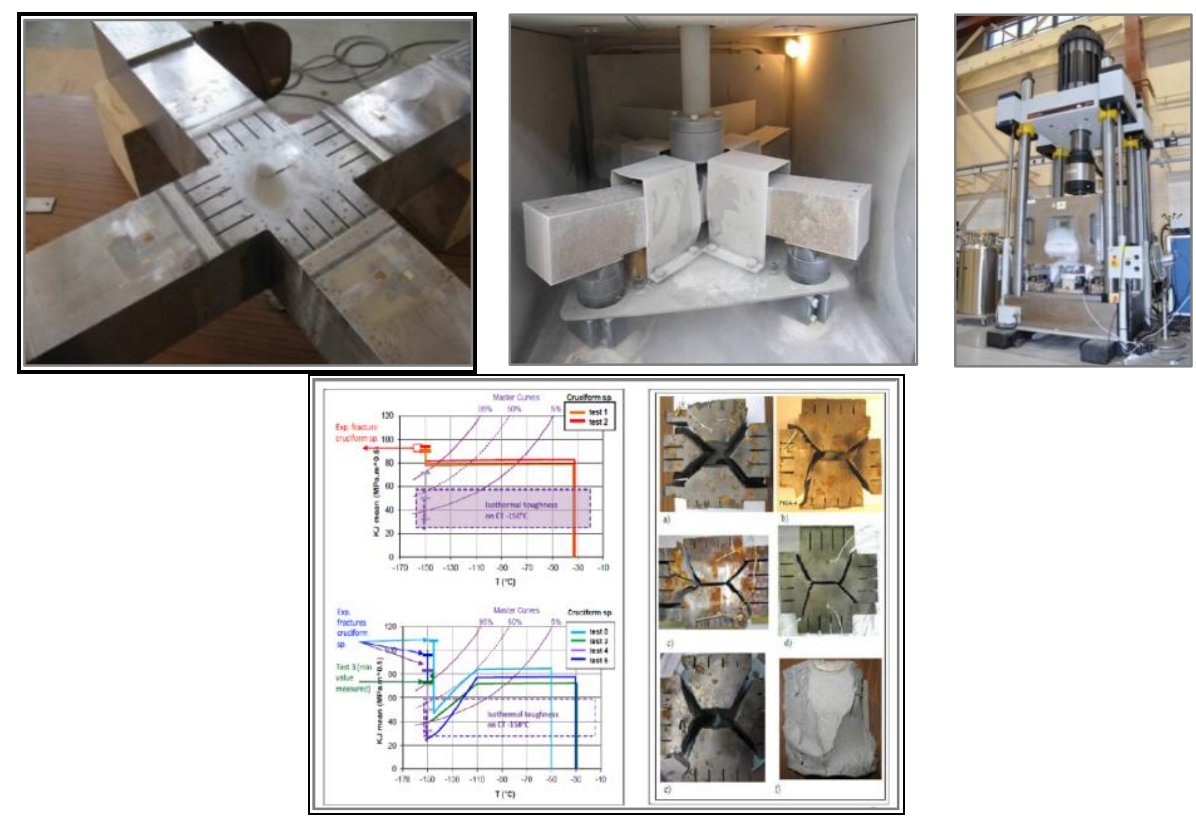

Figure 5. WPS cruciform specimens tested by CEA (18MND5, LCF and LCTF WPS cycles)

\section{Influence of a biaxial loading on WPS: NESC VII SCK-CEN PTS-D disks (18MND5) [3]}

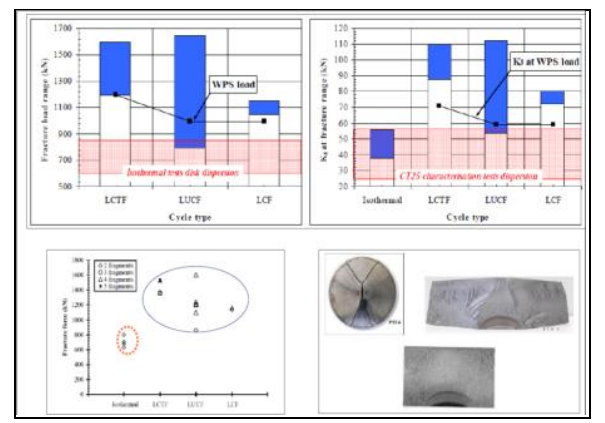

Figure 6. WPS experiments on NESC VII SCK-CEN PTS-D disks (18MND5 steel, LCF, LUCF and LCTF WPS cycles)

Influence of crack geometry on WPS: Uniaxial loading on CEA CTpor specimens (18MND5) [5]
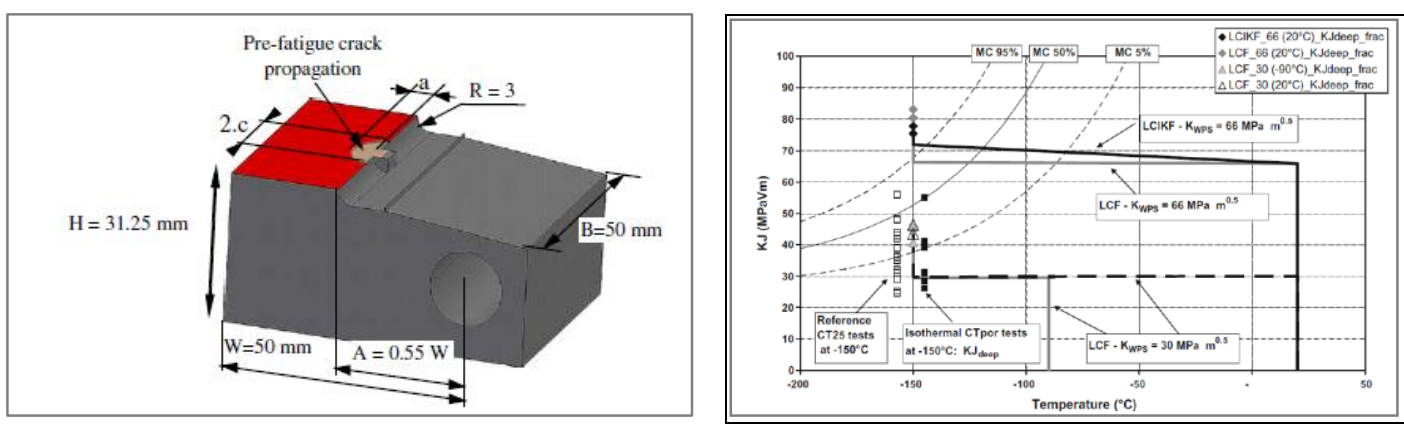

Figure 7. WPS experiments on CEA CTpor specimens (18MND5 steel, $1 / 2$ elliptical crack, LCF and LCIKF WPS cycles) 
Influence of irradiation on WPS: EDF results on irradiated $1 / 2$ T CT specimens $\left(16 M N D 5,5.010^{19} \mathrm{n} / \mathrm{cm}^{2}\right.$ $\left.<\Phi<6.510^{19} \mathrm{n} / \mathrm{cm}^{2}\right)[7][8][9]$
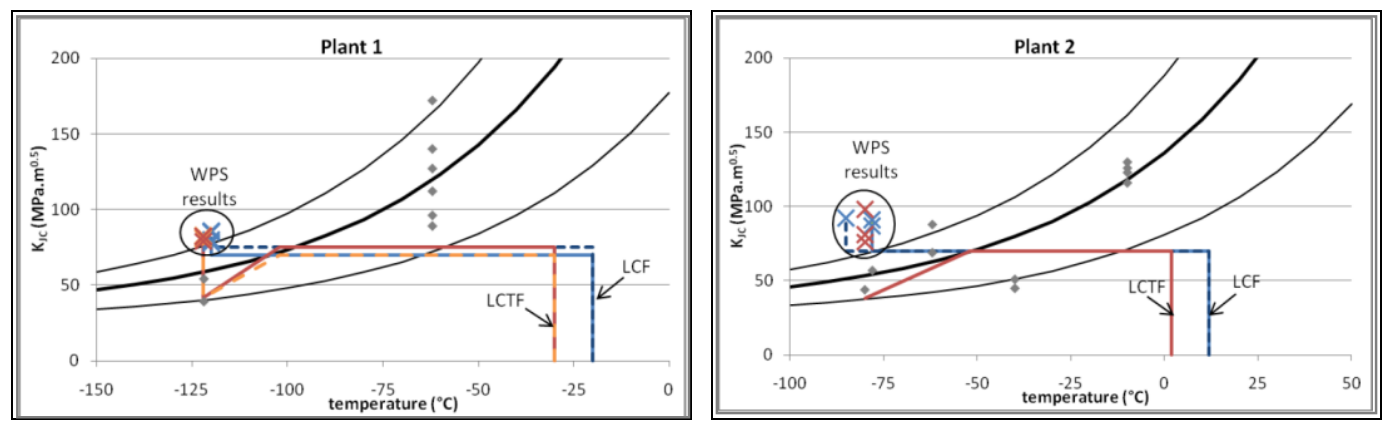

Figure 8. WPS experiments on EDF irradiated 1/2T CT specimens (16MND5 steel, LCF and LCTF WPS cycles)

Influence of high irradiation on WPS: CEA WPS results on highly irradiated $1 / 2$ T CT specimens $\left(16 M N D 5, \Phi \sim 1.410^{20} \mathrm{n} / \mathrm{cm}^{2}\right)[10]$
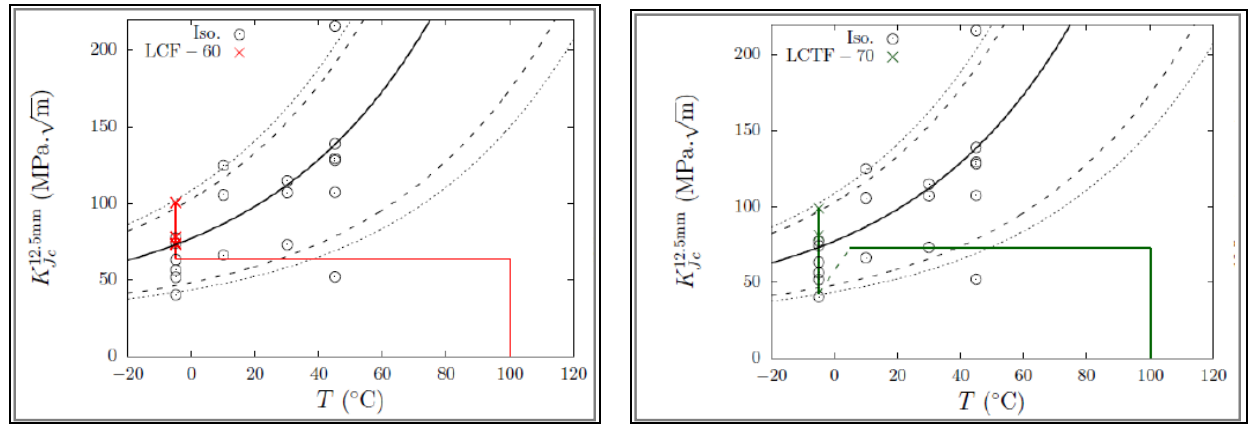

Figure 9. WPS experiments on CEA highly irradiated 1/2T CT specimens (16MND5 steel, LCF and LCTF WPS cycles)

\section{WPS DATABASE}

A specific database has been constituted by EDF ('BDD WPS EDF') to gather all experimental data, including experimental conditions (input data) and fracture results [22]. Figure 10 shows an extract of the database. More than 200 data (test specimens) are thus gathered, to be used for evaluation and validation of WPS analytical and numerical models.

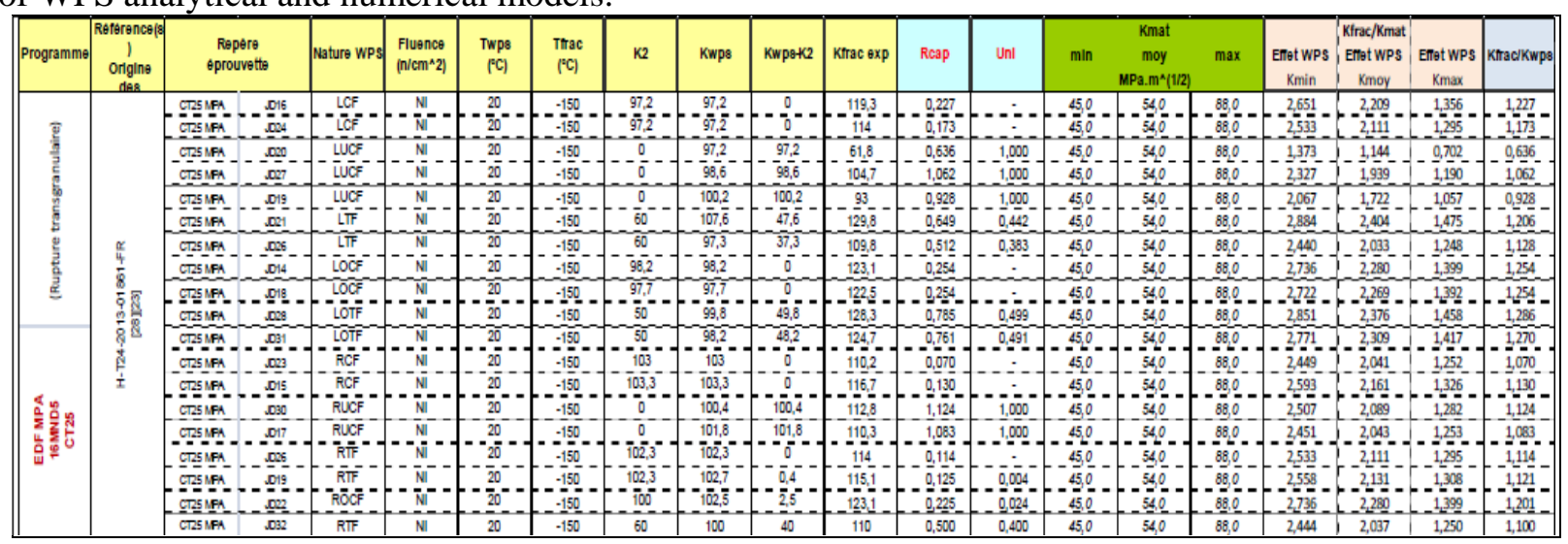

Figure 10. Extract of EDF WPS database 


\section{ACE CRITERION: AN ANALYTICAL MODEL FOR EVALUATION OF FRACTURE TOUGHNESS INDUCED BY WPS}

ACE criterion has been conjointly developed by AREVA, CEA and EDF (ArevaCeaEdf) [11][15]. The objective was to propose a simple engineering criterion for a conservative evaluation of WPS induced effective fracture toughness, for example in RPV integrity assessment.

The basis of the criterion is described in more detail in paper [11] and summarized as follows. The effective fracture toughness induced by WPS $\mathrm{K}_{\mathrm{FRAC}}$ is given by the following equation:

$$
\mathrm{K}_{\mathrm{FRAC}}{ }^{\mathrm{ACE}}=\max \left\{\mathrm{K}_{\mathrm{Ic}} ; \min \left\{\mathrm{K}_{\mathrm{WPS}} ; \mathrm{K}_{2}+\mathrm{K}_{\mathrm{WPS}} / 2\right\}\right\}
$$

depending on three parameters, easily identified (Figure 11):

- $\mathrm{K}_{\mathrm{Ic}}$, original isothermal fracture toughness at the fracture temperature $\mathrm{T}_{\mathrm{FRAC}}$

- $\mathrm{K}_{\mathrm{WPS}}$, level of initial preloading (maximum loading)

- $\mathrm{K}_{2}$, minimum value of loading during the loading path

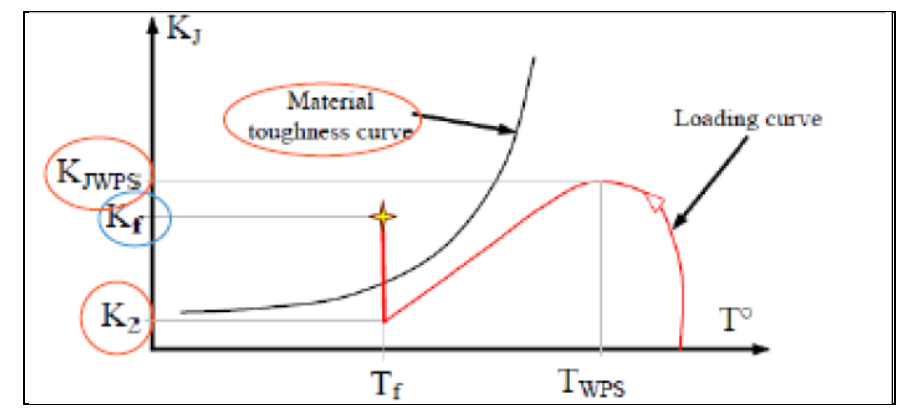

Figure 11. Schematic of WPS loading path and ACE criterion parameters

For the formulation of the criterion, two parameters have been introduced to characterize:

- the relative unloading during the loading path, $\mathrm{U}_{\mathrm{nl}}=\left[\mathrm{K}_{\mathrm{WPS}}-\mathrm{K}_{2}\right] / \mathrm{K}_{\mathrm{WPS}}$

- the relative reloading capability, $\quad \mathrm{R}_{\text {cap }}=\left[\mathrm{K}_{\mathrm{FRAC}}-\mathrm{K}_{2}\right] / \mathrm{K}_{\mathrm{WPS}}$

Starting from initial UJV data, the presentation of experimental results in the $U_{n l}-R_{\text {cap }}$ space (Figure 12) has led to propose a reasonable lower bound, defined in two steps. This lower bound curve was then confirmed on other sets of data [11][15](Figure 12).
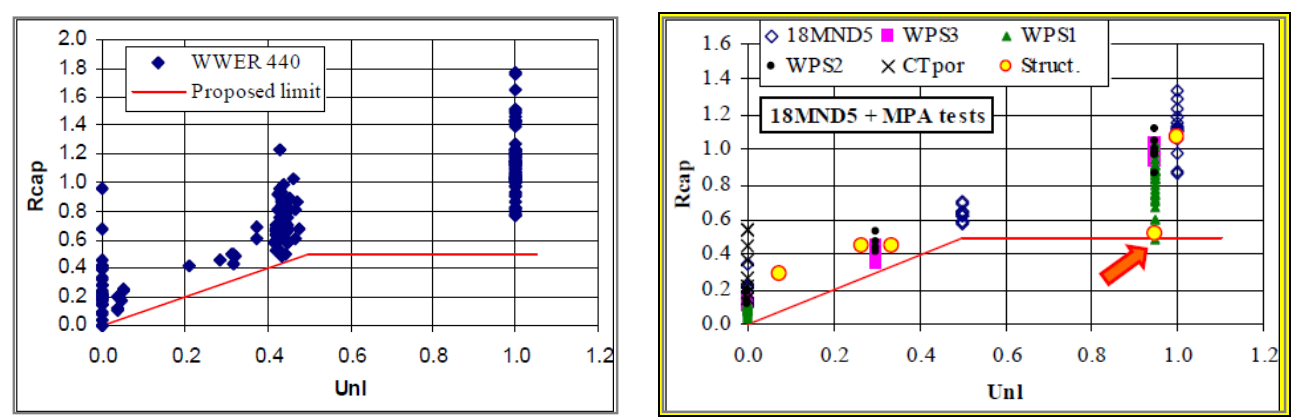

Figure 12. Existence of a lower bound on tests results: NRI data and additional WPS data (18MND5, 17MoV8.4 mod.)

For a limited partial unloading $\left(\mathrm{U}_{\mathrm{nl}}<0.5\right)$, no effect on the reloading capability is observed. The unloading stress field at the crack tip remains quasi-elastic and thus the material is not affected. A supplementary loading is needed ( $\mathrm{R}_{\text {cap }}>\mathrm{U}_{\mathrm{nl}}$ ), so a reasonable envelop can be defined by $\mathrm{R}_{\text {cap }}=\mathrm{U}_{\mathrm{nl}}$. 
For larger partial unloading $\left(0.5<\mathrm{U}_{\mathrm{nl}}<1\right)$, stress unloading is larger and plasticity in compression may occur at the crack tip. Material, residual stress and crack tip blunting are affected, and reloading capability may become lower than unloading $\left(\mathrm{R}_{\text {cap }}<\mathrm{U}_{\mathrm{nl}}\right)$. A reasonable envelop is chosen, $\mathrm{R}_{\text {cap }}=0.5$, is chosen to define the reloading limit.

From these observations, $\mathrm{K}_{\mathrm{FRAC}}{ }^{\mathrm{ACE}}$ can be easily obtained by:

$$
\mathrm{K}_{\mathrm{FRAC}}^{\mathrm{ACE}}=\max \left\{\mathrm{K}_{\mathrm{Ic}} ; \min \left\{\mathrm{K}_{\mathrm{WPS}} ; \mathrm{K}_{2}+\mathrm{K}_{\mathrm{WPS}} / 2\right\}\right\}
$$

\section{EXPERIMENTAL VALIDATION OF ACE CRITERION}

The evaluation of ACE criterion validity is made through the extensive EDF WPS database previously described [15]. A comparison between WPS experimental effective fracture toughness and predictions of ACE criterion is shown on Figure 13 (all materials and limited to irradiated materials). It clearly shows the validity and the conservatism of the ACE criterion towards this large database. Irradiation has no specific influence on the effect of warm pre-stress, even with high fluence $\left(>10^{20} \mathrm{n} / \mathrm{cm}^{2}\right)$.
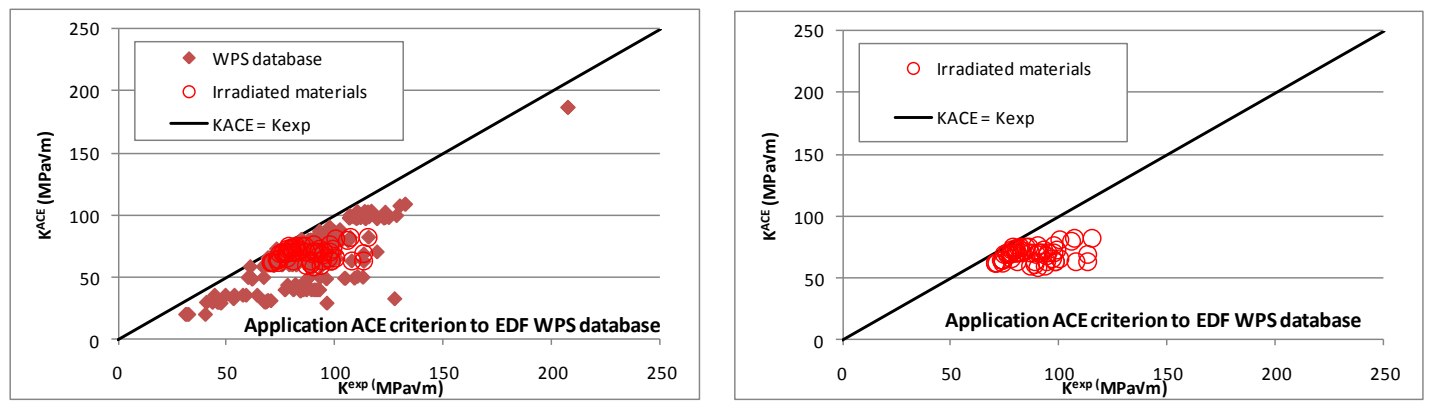

Figure 13. Application of ACE criterion to EDF WPS database (all materials and limited to irradiated materials)

Using the $\mathrm{U}_{\mathrm{nl}}-\mathrm{R}_{\text {cap }}$ space, the lower bound shape is confirmed for all experimental configurations (Figure 14).
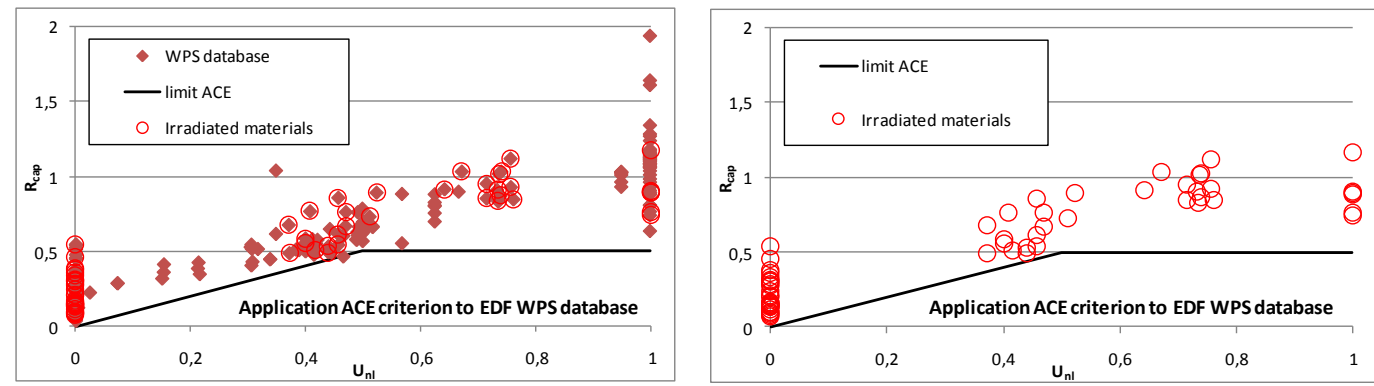

Figure 14. Application of ACE criterion to EDF WPS database (all materials and limited to irradiated materials)

Based on a very significant WPS database covering a large range of experimental conditions, the validity and the conservatism of ACE criterion are clearly demonstrated, through a full range of unloading conditions $(0<\mathrm{Unl}<1)$.

\section{NUMERICAL ANALYSES IN SUPPORT TO WPS AND ACE CRITERION VALIDATION}

In addition to this work, some of the WPS experiments have been interpreted with different kind of approaches, including engineering (Wallin [14], Wallin-NRI, Haigh, ACE) or numerical models, such as local approach to cleavage fracture. 
Most of the numerical analyses have been conducted using the 'modified Beremin' model [13], derived from the original 'Beremin model' [12]. The modification and improvement of the original 'Beremin model' has been specially developed to account non monotonic loading. It thus allows the evaluation of the brittle failure probability of a structure during a complex loading, such as PTS or WPS type loading:

$$
P_{r}=1-e^{\left(-\int\left[\max _{V(u u t, \mathrm{p}(u)>0\}}\left(\frac{\sigma_{I}(u)}{\sigma_{u}(\theta(u))}\right)\right]^{m} \frac{d V}{V_{0}}\right)}
$$

More details on the model are available in paper [13]. This approach requires the identification of two specific parameters $-\mathrm{m}$ and $\sigma_{\mathrm{u}}-$ for the considered material. In practice, $\mathrm{m}$ is fixed constant $(\mathrm{m}=20)$ and $\sigma_{u}$ is temperature dependent, identified in a wide range of temperature. A key point of the 'modified Beremin' model is the notion of 'active plasticity': an increment of plastic deformation is necessary between 2 steps of loading to induce a possible fracture initiation. If it is not the case, there is no possibility of fracture initiation during 2 steps of loading. Some specific 'WPS type' tests have been conducted on CT specimens on un-irradiated (18MND5, 16MND5) and irradiated materials (16MND5) to validate the notion of 'active plasticity' (e.g. [16]).

An illustration of use of local approach to fracture ('modified Beremin' model) is given on Figure 15 (EDF WPS CT tests on irradiated materials) and Figure 16 (CEA experiments on biaxial cruciform specimens):
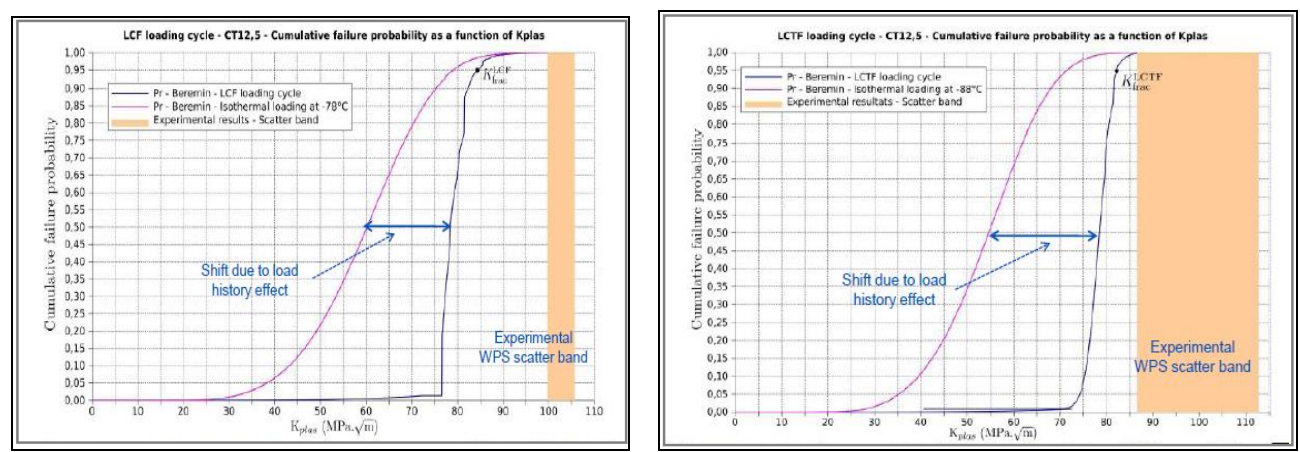

Figure 15. Analysis of EDF WPS CT tests on irradiated material with 'modified Beremin' model (16MND5 steel)
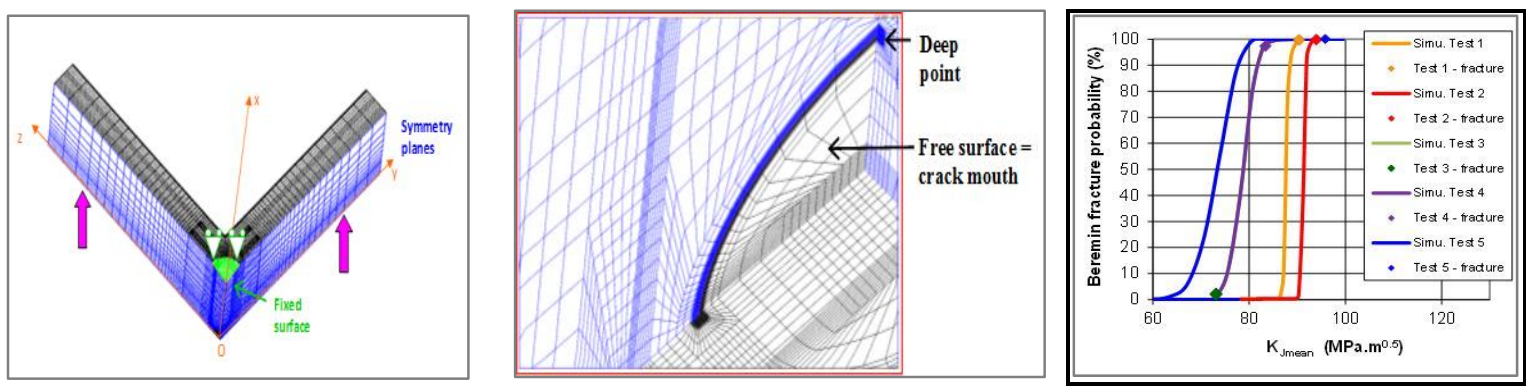

Figure 16. 3D analysis of CEA experiments on biaxial cruciform specimens with 'modified Beremin' model (18MND5 steel)

To validate the ACE criterion, some comparisons between analytical and 'modified Beremin' models are illustrated on Figure 17, without any specific comments. The conservatism of ACE criterion is observed, by comparison of predictions based on local approach. 

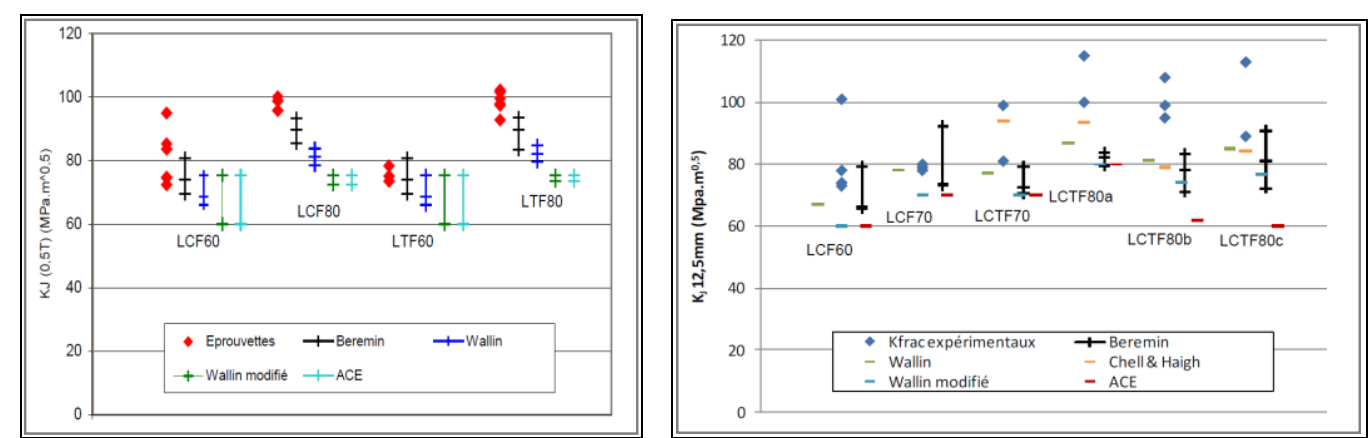

Figure 17. Interpretation of EDF WPS experiments on $1 / 2 \mathrm{~T}$ CT specimens (un-irradiated 16MND5) (LCF and LTF cycles). Comparison between models and experimental results

\section{IMPLEMENTATION OF WPS IN FRENCH RSE-M CODE}

A new appendix has been introduced in 2015 in the French RSE-M code, devoted to in-service operation, dealing with the assessment of defects in reactor pressure vessel [17]. This new appendix reflects the current French practice and introduces a second criteria (option 2) to consider the effect of warm pre-stress in the RPV integrity assessment 5 (Figure 18)(limited to the situations of $3^{\text {rd }}$ and $4^{\text {th }}$ categories).

This appendix is applicable to subclad defects and defects partially in the cladding, and covers nominal, incidental and accidental conditions. The assessment is still based on the comparison between the stress intensity factor and the material fracture toughness (taking into ageing induced by irradiation). If the conventional criterion is not verified for incidental or accidental situations, an alternative criterion is proposed taking into account the effect of warm pre-stress (based on the ACE criterion). More specific details about this new RSE-M appendix are presented in the paper [17].

\begin{tabular}{|c|c|c|}
\hline Level A criteria & Level C criteria & Level D criteria \\
\hline $2^{\text {nd }}$ category transient & $3^{\text {rd }}$ category transient & $4^{\text {th }}$ category transient \\
\hline $\begin{array}{l}\text { Brittle fracture and } \\
\text { transition region } \\
\left(\mathrm{T}_{-}-\mathrm{RT}_{\mathrm{NDT}} \leq 60^{\circ} \mathrm{C}\right) \\
\mathrm{K}_{\mathrm{cp}}\left(2 \mathrm{C}_{\mathrm{A}}, \mathrm{a}_{\mathrm{f}}\right) \leq \mathrm{K}_{\mathrm{Ic}}\end{array}$ & $\begin{array}{l}\text { Brittle fracture and transition region } \\
\qquad \begin{array}{c}\left(\mathrm{T}-\mathrm{RT} \mathrm{NDT}_{\mathrm{NDT}} \leq 60^{\circ} \mathrm{C}\right) \\
\mathrm{K}_{\mathrm{cp}}\left(1.6 \mathrm{C}_{\mathrm{C}}, \mathrm{a}_{\mathrm{f}}\right) \leq \mathrm{K}_{\mathrm{Ic}}\end{array}\end{array}$ & $\begin{array}{l}\text { Brittle fracture and transition region } \\
\qquad\left(\mathrm{T}-\mathrm{RT} \mathrm{T}_{\mathrm{NDT}} \leq 60^{\circ} \mathrm{C}\right) \\
\mathrm{K}_{\mathrm{cp}}\left(\frac{\mathbf{o p t i o n} \mathbf{1}}{\left..2 \mathrm{C}_{\mathrm{D}}, \mathrm{a}_{\mathrm{f}}\right) \leq \mathrm{K}_{\mathrm{Ic}}}\right.\end{array}$ \\
\hline & 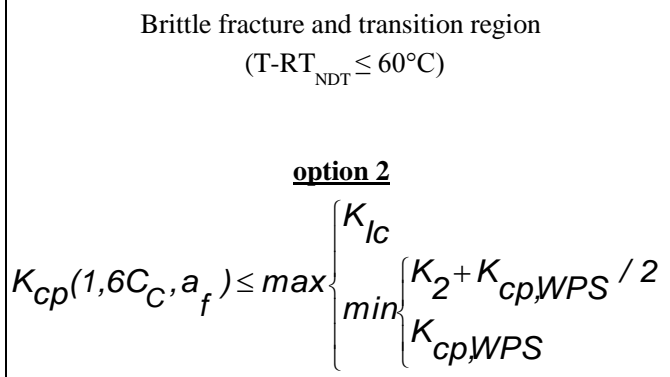 & 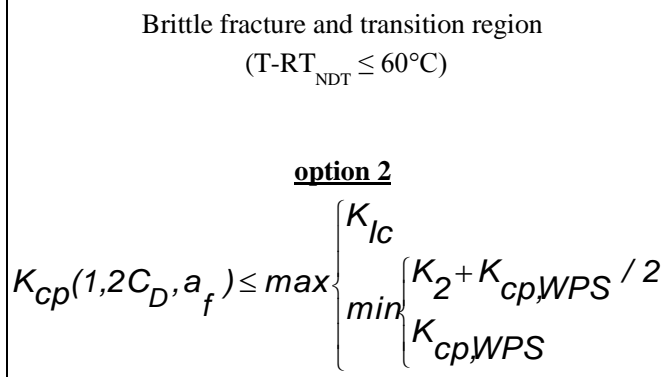 \\
\hline $\begin{array}{c}\text { Ductile region } \\
\left(\mathrm{T}-\mathrm{RT} \mathrm{NDT}_{\mathrm{NDT}} \geq 40^{\circ} \mathrm{C}\right)\end{array}$ & $\begin{array}{c}\text { Ductile region } \\
\left(\mathrm{T}-\mathrm{RT}_{\mathrm{NDT}} \geq 40^{\circ} \mathrm{C}\right)\end{array}$ & $\begin{array}{c}\text { Ductile region } \\
\left(\mathrm{T}-\mathrm{RT}_{\mathrm{NDT}} \geq 40^{\circ} \mathrm{C}\right)\end{array}$ \\
\hline $\mathrm{K}_{\mathrm{cp}}\left(1.5 \mathrm{C}_{\mathrm{A}}, \mathrm{a}_{\mathrm{f}}\right) \leq \mathrm{K}_{\mathrm{Jc}}$ & $\mathrm{K}_{\mathrm{cp}}\left(1.3 \mathrm{C}_{\mathrm{C}}, \mathrm{a}_{\mathrm{f}}\right) \leq \mathrm{K}_{\mathrm{Jc}}$ & $\mathrm{K}_{\mathrm{cp}}\left(1.1 \mathrm{C}_{\mathrm{D}}, \mathrm{a}_{\mathrm{f}}\right) \leq \mathrm{K}_{\mathrm{Jc}}$ \\
\hline
\end{tabular}

Figure 18. Criteria applied to the ferritic steel of the RPV

Margins can be evaluated according 2 criteria: 
- a margin in temperature $\mathrm{M}_{\mathrm{T}}$

- a margin in reloading $\mathrm{M}_{\mathrm{K}}$

The evaluation of these margins - taking into account the WPS effect - is illustrated on Figure 19.
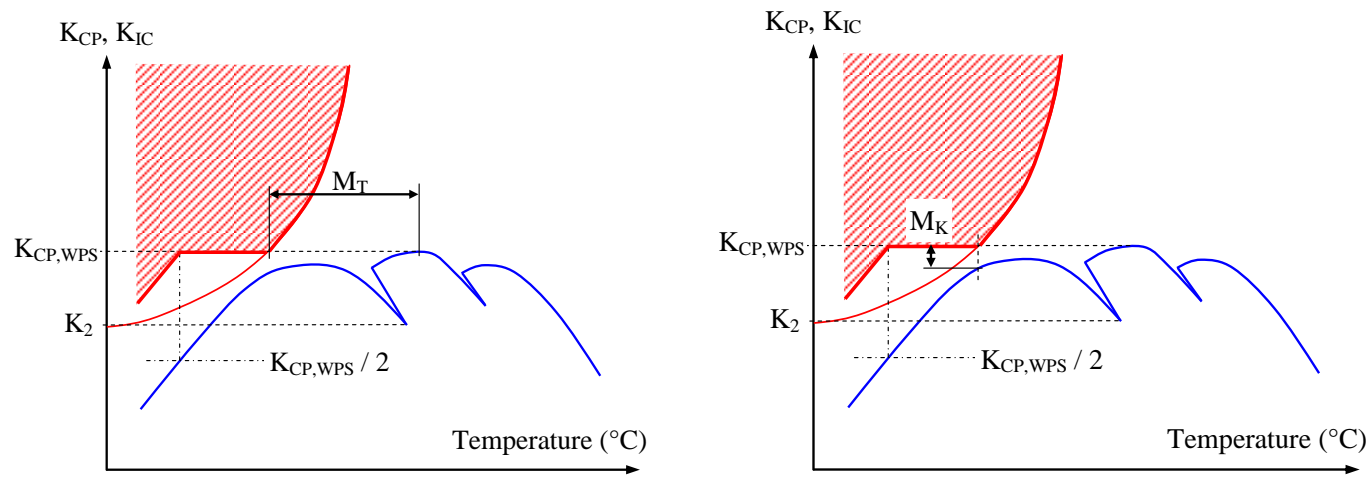

Figure 19. Evaluation of margins $\left(\mathrm{M}_{\mathrm{T}}\right.$ and $\left.\mathrm{M}_{\mathrm{K}}\right)$ taking into account WPS

\section{CONCLUSIONS AND PERSPECTIVES}

A significant work has been conducted in France regarding the applicability of the warm pre-stress effect to the fracture resistance behavior of RPV. Several experimental programs have been conducted and are now achieved, covering a wide range of experimental conditions: un-irradiated and highly irradiated RPV steels, uniaxial or biaxial loading, small, medium and large scale specimens, WPS loading paths, type of loading ...

The beneficial effect of WPS is fully confirmed on the brittle fracture resistance of RPV steels, whatever the experimental conditions:

- the 'conservative principle' is always verified, no fracture occurs during the cooling phase with constant or decreasing loading

- an increase of the material brittle fracture resistance is generally observed in case of reloading at low temperature, compared to the initial isothermal fracture toughness

Based on this work, a specific WPS database has been constituted gathering all experimental data. An analytical criterion - ACE criterion - has been conjointly developed by French organizations (ArevaCeaEdf) for a conservative evaluation of the effective material fracture resistance induced by WPS. Based on the WPS database, the validity and the conservatism of ACE criterion are clearly demonstrated, through a full range of experimental conditions and unloading situations $(0<\mathrm{Unl}<1)$.

In addition to the analytical work, a numerical model derived from the local approach to cleavage fracture has been developed ('modified Beremin' model') and applied for the interpretation of most of the experiments, with successful results. It also confirm the validity - and the conservatism - of the ACE criterion.

The last step is the inclusion of the ACE criterion in the French RSE-M code [1]. A new appendix is now available (2015) in the code taking into account the possibility of using the effect of warm pre-stress in the integrity assessment for the situations of $3^{\text {rd }}$ and $4^{\text {th }}$ categories transients.

\section{ACKNOWLEDGMENT}

The authors thank all colleagues, partners and organizations involved in these $R \& D$ projects devoted to WPS. A specific thank to H. Churier-Bossennec, S. Vidard (EDF SEPTEN) and C. Pagès, N. Jardin (EDF UNIE) for their technical and financial support.

A part of this work has been conducted within the framework of the French R\&D 'Institute Tripartite I3P'. Thanks also for this support. 


\section{REFERENCES}

[1] RSE-M code 'Rules for in-service inspection of nuclear power plant components', AFCEN

[2] D. Moinereau, A. Dahl, S. Chapuliot, D. Lidbury

'SMILE project. The effect of warm pre-stress in RPV assessment: synthesis of experimental results and analyses', ASME PVP 2006 Conference, 23-27 July 2006, Vancouver, Canada

[3] D. Moinereau, S. Chapuliot, S. Marie, C. Jacquemoud

'NESC VII synthesis: a european project for application of WPS in RPV assessment including biaxial loading', ASME PVP 2014 Conference, July 20-24, Anaheim, California, USA

[4] T. Yuritzinn, L. Ferry, S. Chapuliot, P. Mongabure, D. Moinereau, A. Dahl, Ph. Gilles

'Illustration of the WPS benefit through BATMAN test series: tests on large specimens under WPS loading configuration', Engineering Fracture Mechanics, 75 (2008), pp 2191-2007

[5] T. Yuritzinn, L. Ferry, S. Chapuliot, D. Moinereau, A. Dahl, Ph. Gilles

'Warm pre-stressing tests on specimens with semi-elliptical cracks and analysis of the results', Engineering Fracture Mechanics, 77 (2010), 71-83, 2010

[6] C. Jacquemoud, T. Yuritzinn, S. Marie, S. Chapuliot, D. Moinereau, M. Nedelec

'Synthesis of the NESC VII European project: demonstration of warm pre-stressing effect in biaxial loading conditions, ASME PVP 2013 Conference, 14-18 July 2013, Paris, France

[7] G. Chas, E. Molinié, E. Garbay, F. Clémendot, D. Moinereau, H. Churier-Bossennec, C. Pagès

'Fracture toughness of a highly irradiated pressure vessel steel in warm pre-stress loading conditions (WPS)', ASME PVP 2011 Conference, 17-21 July 2011, Baltimore, Maryland, USA

[8] C. Landron, M. Ait-Bachir, D. Moinereau, E. Molinié, E. Garbay

'Warm pre-stress experiments on highly irradiated reactor pressure vessel steel', Fontevraud 8 Symposium, 14-18 September 2014, Avignon, 2014

[9] D. Moinereau, C. Landron, M. Ait Bachir, E. Garbay

'Effect of warm pre-stress on highly irradiated pressure vessel steel', ASME PVP 2014 Conference, July 20-24, Anaheim, California, USA

[10] J. Hure, C. Vaille, P. Wident, D. Moinereau, C. Landron, S. Chapuliot, C. Benhamou, B. Tanguy

'Warm PreStress effect on highly irradiated reactor pressure vessel steel', Journal of Nuclear Materials 00 (2015) 1-22, 2015

[11] S. Chapuliot, J.P. Izard, D. Moinereau, S. Marie

'WPS criterion proposition based on experimental data base interpretation', Fontevraud 7 Symposium, 2630 September 2010, Avignon, 2010[19] F. Mudry

[12] F. Mudry

'A local approach to cleavage fracture', Proceedings of the International Seminar on local approach of fracture, 3-4-5 June 1986, Moret-sur-Loing, France

[13] R. Masson et al

'A modified Beremin model to simulate the warm pre-stress effect', Journal of Nuclear Engineering and Design, 216, pp 27-42, 2002

[14] K. Wallin

'Master curve implementation of the warm pre-stress effect', Engineering Fracture Mechanics, 70, 25872602, 2003

[15] D. Moinereau, C. Landron, S. Chapuliot, S. Marie

'Validation of ACE analytical criterion for warm pre-stress evaluation in RPV integrity assessment', ASME PVP 2015 Conference, July 19-23, Boston, Massachussets, USA

[16] C. Jacquemoud, S. Marie, M. Nédélec

'Evaluation of the active plasticity hypothesis as a relevant justification of warm pre stressing effect', Engineering Fracture Mechanics 104 (2013) 16-28

[17] S. Marie, S. Chapuliot, D. Moinereau, M. Ait Bachir, C. Jacquemoud, B. Tanguy

'Introduction of warm pre-stress concept in French RPV structural integrity assessment', Part II, ASME PVP 2016 Conference, July 17-21, Vancouver, British Columbia, Canada 